\title{
History of Repeated Bleeding from Intact Skin and Mucous Membranes: A Quiz
}

Saskia B. WORTMANN ${ }^{1,2}$, Tobias WELPONER ${ }^{1}$ and Martin LAIMER ${ }^{1}$

${ }^{1}$ University Children's Hospital, Paracelsus Medical University (PMU), Salzburg, Austria and ${ }^{2}$ Radboud Center for Mitochondrial Medicine, Department of Pediatrics, Amalia Children's Hospital, Radboudumc, Nijmegen, The Netherlands. E-mail: s.wortmann@salk.at

A 12-year-old girl presented with a 3-month history of episodes of well-defined bloody oozing from intact skin (face, forehead, trunk, upper, lower limbs) and mucosal membranes (mouth, tongue, conjunctiva) (Fig. 1). These attacks occurred at varying sites and preferentially at evening or during sleep. They were occasionally preceded by a subtle burning and tingling sensation and resolved spontaneously after seconds to few minutes. The bloodstain was easily wiped off, revealing normal appearing, non-traumatized skin underneath. Samples of the secretion, appearing clearer and more fluid than blood, contained morphologically inconspicuous erythrocytes. The events became more frequent (to several times a day and during the night, disturbing her sleep) and widespread, with ongoing course, posing a great psychological burden. She additionally developed weekly bouts of headaches, hypaesthesia of the left upper extremity, nausea and abdominal pain. No psychological, physical (stress), infectious, artificial and medication triggers, nor symptoms of arterial hypertension, gastrointestinal, blood, coagulation or psychiatric disorders could be determined.

What is your diagnosis? See next page for answer.

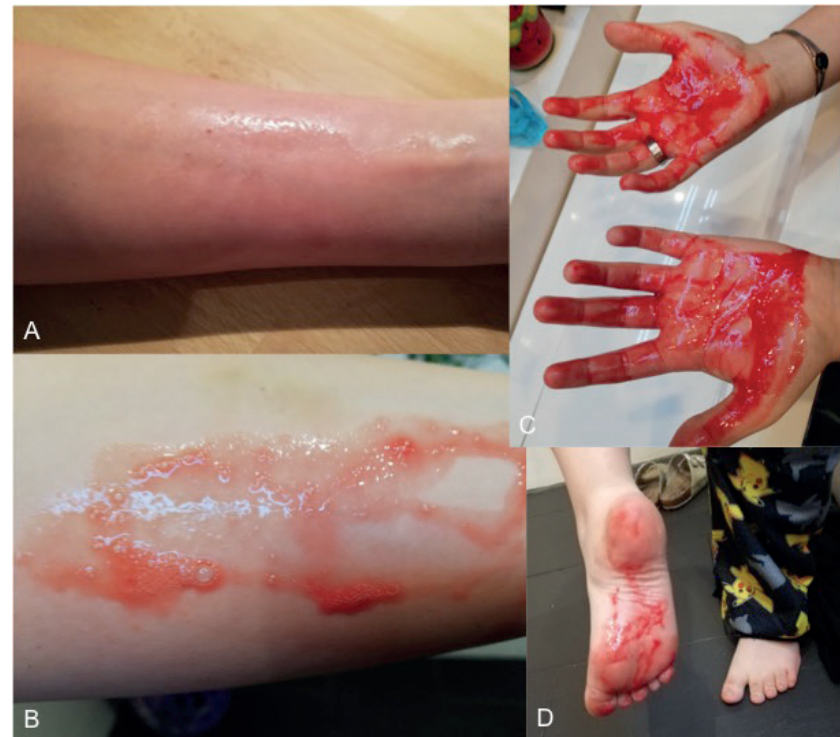

Fig. 1 (A-D). Bloody oozing from upper and lower extremities. The blood could be wiped off easily, revealing intact skin underneath. 


\section{ANSWERS TO QUIZ}

\section{History of Repeated Bleeding from Intact Skin and Mucous Membranes: A Commentary}

Acta Derm Venereol 2021; 101: adv00592.

\section{Diagnosis: Haematohidrosis}

The features of this case are consistent with haematohidrosis, a very rare phenomenon mostly affecting the craniofacial area of female children (1). The pathomechanism remains unclear (1). Some authors proposed dermal defects to lead to blood-filled spaces that would exude into follicular canals or directly to the skin surface (2). Nuclear magnetic resonance (NMR) analysis in a 6-year-old patient further revealed that fluid samples had a metabolomic profile similar to eccrine sweat, containing constituents derived from both blood and sweat (1). The previously reported response to propranolol and the association with stress were suggested to reflect a pathognomonic adrenergic stimulation and vasoconstriction in sweat glands and small blood vessels, leading to blood extravasation (3).

Although self-limited resolution is commonly described, various therapeutic approaches to address quality of life and issues of stigmatization have been reported in the literature, including psychotherapy, relaxation therapies, anxiolytic drugs, antidepressants, anticholinergic agents, and beta-blockers $(1,4)$. In the current case, treatment with beta-blockers (propranolol $3 \times 1.5 \mathrm{mg} / \mathrm{kg} / \mathrm{day}$ ) reduced the frequency of the bloody oozings to once a month and resulted in the disappearance of all other signs and symptoms.

The authors have no conflicts of interest to declare.

\section{REFERENCES}

1. Hansson K, Johansson EK, M Albåge M, Ballardini N. Paediatric haematohidrosis: an overview of a rare but clinically distinct condition. Acta Paediatr 2019; 108: 1023-1027.

2. Deshpande $M$, Indla V, Kumar V, Reddy IR. Child who presented with hematohidrosis (sweating blood) with oppositional defiant disorder. Indian J Psychiatr 2014; 56: 289-291.

3. Wang Z, Yu Z, Su J, Cao L, Zhao X, Bai X, et al. A case of hematidrosis successfully treated with propranolol. Am J Clin Dermatol 2010; 11: 440-403.

4. Tirthani K, Sardana K, Mathachan SR. Hematohidrosis of the mid-face and hands treated with oral oxybutynin. Pediatr Dermatol 2021; 38: 962-963. 